\title{
Drug-induced movement disorders
}

Stephen R Duma

John Morris movement

disorders fellow?

Clinical lecturer ${ }^{2}$

\section{Victor SC Fung}

Director

Clinical associate professor ${ }^{2}$

1 Movement Disorders Unit, Department of Neurology, Westmead Hospital, Sydney 2 Sydney Medical School, University of Sydney

\section{Keywords}

dystonia, neuroleptic malignant syndrome, parkinsonism, serotonin syndrome, tardive dyskinesia

Aust Prescr 2019;42:56-61 https://doi.org/10.18773/ austprescr.2019.014

\section{SUMMARY}

Many therapeutic and illicit drugs can cause movement disorders. Antipsychotics and antiemetics are most commonly implicated.

The time of onset of the movement disorder may be acute, subacute, or chronic. The severity can range from mild to severe and life-threatening.

Early recognition of a drug-induced movement disorder is essential to allow for prompt intervention. This includes stopping the offending drug, supportive care, and sometimes other pharmacological treatment.

\section{Introduction}

Both therapeutic and illicit drugs can cause neurological adverse effects, including movement disorders. The most common causes of drug-induced movement disorders are dopamine receptor blocking drugs, including antipsychotics and antiemetics (Table 1). Drug-induced movement disorders can range from tremors to life-threatening syndromes. They can be classified chronologically based on the time of onset after drug ingestion, as acute, subacute or tardive.

\section{Acute disorders}

Acute drug-induced movement disorders occur within minutes to days of drug ingestion. They include akathisia, tremor, neuroleptic malignant syndrome, serotonin syndrome, parkinsonism-hyperpyrexia disorder and acute dystonic reactions. ${ }^{1-4}$

\begin{abstract}
Akathisia
Akathisia is a common, but often under-recognised, drug-induced movement disorder that can occur as an acute, subacute or tardive reaction. It is a sense of internal restlessness, irritability and tension without necessarily manifesting with physical signs, unlike restless legs syndrome which is typically more severe and worse at night. Akathisia has been reported with dopamine receptor blockers, selective serotonin reuptake inhibitors (SSRIs), antiepileptic drugs, and cocaine. It can occur either after starting a dopamine receptor blocker, dose escalation, or when switching to an alternative drug.
\end{abstract}

Akathisia often improves following cessation of the offending drug. Anticholinergics, beta blockers, benzodiazepines, amantadine, mirtazapine and clonidine have also been used with varying efficacy and with minimal evidence.

\section{Tremor}

Drug-induced tremor is typically postural or kinetic, or both. It is symmetrical and occurs acutely following drug ingestion or dose escalation. Exceptions include tremor secondary to valproate, which can appear at therapeutic or during stable treatment, or, rarely, tardive tremor. Tremor can occur secondary to many drugs, including SSRIs, lithium, tricyclic antidepressants, antiepileptics (particularly valproate), bronchodilators, amiodarone and immunosuppressives. Another underlying aetiology, such as Parkinson's disease, essential tremor or hyperthyroidism, needs to be excluded.

Management consists of altering the dose of, or if possible stopping, the offending drug, or switching to an alternative drug. Should the offending drug need to be continued, discuss the risks of the adverse effects versus the benefits of continuing to ensure the patient is informed. If the drug is continued, drugs typically used for essential tremor (for example, propranolol) can occasionally be beneficial.

\section{Serotonin syndrome}

Serotonin syndrome occurs secondary to drugs that increase serotonin activity (Table 1). Like neuroleptic malignant syndrome, it can be lifethreatening, but milder forms can occur. Clinical characteristics include:

- altered mental status

- $\quad$ signs of central nervous system hyperexcitability

- movement disorders, including myoclonus, tremor, akathisia

- hyperreflexia, clonus, spasticity or rigidity, seizures

- autonomic instability, including mydriasis, fever and tachycardia. 


\section{Table 1 Drug-induced movements disorders}

\begin{tabular}{ll} 
Movement disorder & Implicated drugs \\
\hline Akathisia & Dopamine receptor blocking drugs \\
& Selective serotonin reuptake inhibitors \\
& Antiepileptics \\
Tremor & Selective serotonin reuptake inhibitors \\
& Lithium \\
& Tricyclic antidepressants \\
& Antiepileptics (e.g. valproate) \\
& Bronchodilators \\
& Amiodarone \\
Immunosuppressive drugs (tacrolimus, ciclosporin)
\end{tabular}

Serotonin syndrome (usually due to overdose or combinations of serotoninergic drugs)

Acute dystonic reaction

Neuroleptic malignant syndrome
Selective serotonin reuptake inhibitors

Serotonin noradrenaline reuptake inhibitors

Tricyclic antidepressants

Monoamine oxidase inhibitors

Lithium

Linezolid

Opioids (pethidine, tramadol, propentadol)

Antiepileptics (valproate, lamotrigine)

St John's wort

Dopamine receptor blocking drugs (e.g. antipsychotics, metoclopramide)

Selective serotonin reuptake inhibitors

Opioids

Methylphenidate

Rivastigmine

Albendazole

Gabapentin

Cetirizine

Foscarnet

Quinine

Propofol

Sevoflurane

Antipsychotics (e.g. haloperidol, fluphenazine, chlorpromazine)

Prochlorperazine

Metoclopramide

Droperidol

Promethazine

Tetrabenazine

Lithium

Dopamine receptor blocking drugs (e.g. antipsychotics)

Calcium channel antagonists (e.g. flunarizine, cinnarizine)

Antiepileptics (e.g. phenytoin, valproate, levetiracetam)

Antidepressants (e.g. selective serotonin reuptake inhibitors, monoamine oxidase inhibitors)

Lithium

Chemotherapeutic drugs (e.g. cystosine arabinoside, cyclophosphamide, vincristine, adriamycin, doxorubicin, paclitaxel, etoposide) Immunosuppressive drugs (e.g. ciclosporin, tacrolimus)

Toxins (e.g. 1-methyl-4-phenyl-1,2,3,6-tetrahydropyridine (MPTP), organophosphate pesticides, manganese, methanol, cyanide, carbon monoxide and carbon disulphide)

\section{Antipsychotics}

Antiemetics (e.g. metoclopramide) 
The altered mental status, autonomic instability, and spasticity or rigidity with raised creatine kinase, overlap with neuroleptic malignant syndrome. In serotonin syndrome the onset is hyperacute, within hours rather than days, and the signs of central nervous system hyperexcitability are more prominent.

\section{Management}

Discontinuation of the offending drugs and supportive care (which may include intensive care) are first-line in treating serotonin syndrome. Cyproheptadine may be given in less severe cases and, if a response is observed, it should be continued until symptoms resolve. ${ }^{5}$ Benzodiazepines or other 5-hydroxytryptamine 2 receptor antagonists (such as chlorpromazine or olanzapine) have been used in severe cases. ${ }^{4,5}$

\section{Parkinsonism-hyperpyrexia disorder}

Parkinsonism-hyperpyrexia disorder, also known as akinetic crisis, is a rare but potentially fatal complication of Parkinson's disease. It involves a syndrome of significantly worsening parkinsonism (with or without encephalopathy), hyperpyrexia, autonomic instability and elevated creatine kinase. ${ }^{4-6}$ The disorder is most commonly seen in patients with Parkinson's disease who have reduced or stopped their antiparkinsonian drugs. It can also be precipitated by an infection or other metabolic disturbance. The clinical features overlap with neuroleptic malignant syndrome. It is also important to exclude alternative causes, including an underlying infection, metabolic abnormalities, or stroke. Recovery can take hours to weeks following treatment.

\section{Management}

The mainstay of treatment includes resuming antiparkinsonian drugs, usually via nasogastric tube because of the dysphagia resulting from severe parkinsonism. Intermittent apomorphine injections or a continuous infusion may be required in moderatesevere cases.

\section{Acute dystonic reactions}

Acute dystonic reactions most commonly occur in younger patients soon after taking to dopamine receptor blocking drugs, including antiemetics (e.g. metoclopramide or prochlorperazine) and antipsychotics. Acute sustained dystonic spasm of craniocervical muscles is typical, but oculogyric crises, truncal spasm causing opisthotonos, or limb dystonia can also occur. Acute laryngeal dystonia can be lifethreatening due to airway obstruction and requires emergency medical care.

\section{Management}

Stop the offending drug, and give an intravenous or intramuscular anticholinergic drug (such as benzatropine or trihexyphenidyl (benzhexol) hydrochloride). As the injectable drug has a short half-life it is followed by a short course of oral anticholinergic drugs. ${ }^{4,5,7}$ Benzodiazepines have also been used. It is important to avoid the offending drug in the future due to the risk of a recurrent dystonic reaction. Educate the patient regarding this risk.

\section{Neuroleptic malignant syndrome}

Neuroleptic malignant syndrome is a potentially life-threatening reaction to typical and atypical antipsychotic drugs and other dopamine receptor blocking drugs, including tetrabenazine, lithium and antiemetics such as metoclopramide. Delphi consensus diagnostic criteria ${ }^{8}$ have been recently validated. ${ }^{9}$ These criteria include:

- exposure to a dopamine antagonist, or dopamine agonist withdrawal, within the past 72 hours

- hyperthermia $\left(>38^{\circ} \mathrm{C}\right.$ on at least two occasions)

- rigidity

- altered mental status

- elevated creatine kinase

- autonomic instability (including hypermetabolism, i.e. tachycardia and tachypnoea)

- negative investigations for an alternative cause.

In addition to the elevated creatine kinase, laboratory investigations usually find leucocytosis, abnormal electrolytes, renal impairment, abnormal liver function tests, and altered coagulation studies. Milder cases without all the clinical features can occur.

There are a number of differential diagnoses, including serotonin syndrome, and specialist assessment is required. For example, serotonin syndrome tends to occur more acutely than neuroleptic malignant syndrome. There is rigidity in neuroleptic malignant syndrome whereas myoclonus, hyperreflexia with clonus, and mydriasis are more common in serotonin syndrome.

\section{Management}

If neuroleptic malignant syndrome is suspected, acute hospital admission is warranted. Management involves immediate cessation of the offending drugs, supportive care (which includes intensive care if severe), and giving a dopaminergic drug, usually bromocriptine. Subcutaneous apomorphine injections have also been used. Benzodiazepines can be used to reduce rhabdomyolysis and improve rigidity. 
The syndrome typically plateaus and improves within 2-3 weeks of onset. Bromocriptine should therefore be continued for several weeks to ensure the syndrome has completely subsided. Consideration about restarting an antipsychotic requires a specialist psychiatric opinion.

\section{Subacute disorders}

Subacute drug-induced movement disorders occur within days to weeks of drug ingestion. Some of the syndromes listed in Table 1 can develop subacutely. They usually respond to cessation of the offending drug.

\section{Parkinsonism}

Drug-induced parkinsonism is typically characterised by bradykinesia, rigidity and postural instability. It is the second commonest cause of parkinsonism after idiopathic Parkinson's disease. Various drugs have been associated with parkinsonism (see Table 1).

In contrast to idiopathic Parkinson's disease, drug-induced parkinsonism usually presents as a symmetrical akinetic rigid syndrome which develops over days to weeks to months following ingestion of the offending drug. Additionally, there is a poor response to typical antiparkinsonian drugs, including levodopa, dopamine agonists and anticholinergic drugs. Cessation of the offending drug usually results in complete resolution of the disorder.

Additionally, toxins can cause parkinsonism. These include 1-methyl-4-phenyl-1,2,3,6-tetrahydropyridine (MPTP), organophosphate pesticides, manganese, methanol, cyanide, carbon monoxide, and carbon disulphide. Unlike the drugs, toxins are often associated with irreversible structural damage to the basal ganglia visible on MRI.

\section{Levodopa-induced dyskinesia}

Levodopa-induced dyskinesia is a common cause of dyskinesia in individuals with Parkinson's disease. It occurs due to the relationship between dopaminergic loss and the resultant response to levodopa, rather than being due to excess levodopa ingestion only. Risk factors for developing dyskinesia include young age at onset of Parkinson's disease, higher levodopa dose, low body weight, and more severe disease. ${ }^{10}$ A careful history is vital in establishing a pattern to the timing and duration of dyskinesias, which can then assist in altering the levodopa dose.

Depending on the duration of dyskinesia, the levodopa dose can usually be reduced to a lower dose which still maintains efficacy. It is worth noting that mild dyskinesias are often not bothersome to the individual and do not interfere with their function, therefore a change in levodopa dose may not be required. Amantadine can also be used to manage levodopa-induced dyskinesias. Referral is recommended for patients with late-stage disease for consideration of device-assisted therapy.

\section{Tardive disorders}

Tardive drug-induced movement disorders occur either during exposure or within weeks of stopping a drug and are present for at least one month.,11-14 The minimum duration of exposure to the drug is three months, or one month in adults aged over 60 years. The most commonly implicated drugs include antipsychotics, antiemetics (metoclopramide and prochlorperazine) and some calcium channel antagonists with dopamine receptor blocking properties (cinnarizine and flunarizine).

Tardive movement disorders include dyskinesias (typically orobuccolingual), stereotypies, akathisia, dystonia (focal, segmental or generalised), myoclonus, tremor and tics. Additionally, tardive parkinsonism may be experienced. Withdrawal-emergent dyskinesia can occur on abrupt cessation of long-term antipsychotic treatment, particularly in children. The dyskinesia improves on resuming the drug. The dose can then be gradually reduced.

\section{Management}

No good evidence exists regarding the management of tardive drug-induced movement disorders. ${ }^{15}$ Treatment usually consists of withdrawing the offending drug, and a trial of a combination of drugs. Clonazepam has been effective particularly for myoclonus. Resuming the offending drug or changing to an atypical antipsychotic is sometimes required. ${ }^{16}$ In patients with a chronic psychotic disorder clozapine is preferred. Most recently, vesicular monoamine transporter 2 inhibitors deutetrabenazine and valbenazine have been proposed as treatment options. ${ }^{17,18}$ Other oral drugs have been tried, including tetrabenazine, amantadine and propranolol.

Antioxidants, including vitamin $\mathrm{E}$, vitamin $\mathrm{B}_{6}$ and Ginkgo biloba, have also been studied. Vitamin E had conflicting results, while vitamin $\mathrm{B}_{6}$ and Ginkgo biloba are probably useful in treating tardive movement disorders. ${ }^{17,18}$ Caution is needed with Ginkgo biloba because of its antiplatelet effects, especially in patients taking antiplatelet drugs or anticoagulants. Anticholinergic drugs to prevent, or reduce the severity of, drug-induced movement disorders have been suggested, however there is no evidence to support this.

Botulinum toxin injections can be effective for focal manifestations of tardive dystonia. ${ }^{19}$ Deep brain stimulation, targeting the globus pallidus, can be highly effective in severe cases. ${ }^{20}$ 


\section{SELF-TEST}

\section{QUESTIONS}

\section{True or false?}

1. Antiemetics can cause neuroleptic malignant syndrome.

2. Levodopa should be stopped if a patient with Parkinson's disease develops parkinsonismhyperpyrexia disorder.

Answers on page 79

\section{Illicit drugs}

Movement disorders secondary to illicit drugs are usually acute and self-limiting, ${ }^{4,21}$ but can occasionally be life-threatening (Table 2). Cocaine blocks dopamine reuptake thereby increasing dopaminergic drive. Amphetamines cause more widespread catecholaminergic stimulation, but chronic use results in dopamine depletion, and is possibly associated with nigral damage. ${ }^{22}$ 3,4-methylenedioxymethamphetamine (MDMA) is known to cause parkinsonism and a syndrome similar to serotonin syndrome.

While the movement disorder usually occurs following drug ingestion, it can also occur during the withdrawal phase. Typically, it subsides on cessation of the drug, but can last for months. No specific treatment exists for movement disorders caused by illicit drug use.

\section{Conclusion}

Movement disorders are a common, and at times life-threatening, adverse effect of many drugs, most commonly dopamine receptor blocking drugs. Patients are often on combinations of drugs that may cause more than one movement disorder, thereby making it challenging to identify the culprit drug. The diagnosis requires knowledge of the typical movement disorders and the syndromes that can occur with different drug classes, and their typical time course. This is important because the most imperative therapeutic intervention for most drug-induced movement disorders is stopping the offending drug, with or without supportive or other pharmacological treatment. $<$

Victor Fung receives a salary from NSW Health, has received unrestricted research grants from Abbvie and Merz, is on advisory boards and has received travel grants from Abbvie, Allergan, Cavion, Ipsen, Merz, Praxis, Seqirus, Stada, Teva and UCB, and receives royalties from Health Press.

Dr Duma has received a speaker honorarium from UCB.

\section{Table 2 Illicit drugs and associated movement disorders}

\begin{tabular}{ll}
\hline Drug & Movement disorder \\
\hline Cocaine & Choreoathetosis (chorea and dystonia, also known as 'crack dancing') \\
& Stereotypies \\
& Tremor \\
& Myoclonus \\
& Punding (purposeless, repetitive behaviours) \\
Amphetamines & Tremor \\
& Dystonia \\
& Choreoathetosis \\
& Orolingual dyskinesia \\
3,4-methylenedioxymethamphetamine (MDMA) & Serotonin syndrome \\
& Parkinsonism \\
Opioids & Myoclonus \\
\hline
\end{tabular}

\section{REFERENCES}

1. Claxton KL, Chen JJ, Swope DM. Drug-induced movement disorders. J Pharm Pract 2007;20:415-29. https://doi.org/ 10.1177/0897190007310514

2. Malek N, Baker MR. Common toxidromes in movement disorder neurology. Postgrad Med J 2017;93:326-32. https://doi.org/10.1136/postgradmedj-2016-134254

3. Caroff SN, Campbell EC. Drug-induced extrapyramidal syndromes: implications for contemporary practice. Psychiatr Clin North Am 2016;39:391-411. https://doi.org/ 10.1016/j.psc.2016.04.003

4. Burkhard PR. Acute and subacute drug-induced movement disorders. Parkinsonism Relat Disord 2014;20 Suppl 1:S108-12. https://doi.org/10.1016/S1353-8020(13)70027-0

5. Kipps CM, Fung VS, Grattan-Smith P, de Moore GM Morris JG. Movement disorder emergencies. Mov Disord 2005;20:322-34. https://doi.org/10.1002/mds.20325
6. Newman EJ, Grosset DG, Kennedy PG. The parkinsonismhyperpyrexia syndrome. Neurocrit Care 2009;10:136-40. https://doi.org/10.1007/s12028-008-9125-4

7. Marano M, di Biase L, Salomone G, Di Santo A, Montiroli A, Di Lazzaro V. The clinical course of a drug-induced acute dystonic reaction in the emergency room. Tremor Other Hyperkinet Mov (N Y) 2016;6:436. https://doi.org/10.7916/D87P8ZS1

8. Gurrera RJ, Caroff SN, Cohen A, Carroll BT, DeRoos F, Francis A, et al. An international consensus study of neuroleptic malignant syndrome diagnostic criteria using the Delphi method. J Clin Psychiatry 2011;72:1222-8. https://doi.org/10.4088/JCP.10m06438

9. Gurrera RJ, Mortillaro G, Velamoor V, Caroff SN. A validation study of the international consensus diagnostic criteria for neuroleptic malignant syndrome. J Clin Psychopharmacol 2017;37:67-71. https://doi.org/10.1097/JCP.0000000000000640 
10. Warren Olanow C, Kieburtz K, Rascol O, Poewe W, Schapira AH, Emre M, et al.; Stalevo Reduction in Dyskinesia Evaluation in Parkinson's Disease (STRIDE-PD) Investigators. Factors predictive of the development of levodopainduced dyskinesia and wearing-off in Parkinson's disease. Mov Disord 2013;28:1064-71. https://doi.org/10.1002/ mds. 25364

11. Aquino CC, Lang AE. Tardive dyskinesia syndromes: current concepts. Parkinsonism Relat Disord 2014;20 Suppl 1:S113-7. https://doi.org/10.1016/S1353-8020(13)70028-2

12. Cornett EM, Novitch M, Kaye AD, Kata V, Kaye AM. Medication-induced tardive dyskinesia: a review and update. Ochsner J 2017;17:162-74.

13. Frei K, Truong DD, Fahn S, Jankovic J, Hauser RA. The nosology of tardive syndromes. J Neurol Sci 2018;389:10-6. https://doi.org/10.1016/j.jns.2018.02.008

14. Savitt D, Jankovic J. Tardive syndromes. J Neurol Sci 2018;389:35-42. https://doi.org/10.1016/j.jns.2018.02.005

15. Bhidayasiri R, Fahn S, Weiner WJ, Gronseth GS, Sullivan KL, Zesiewicz TA; American Academy of Neurology. Evidencebased guideline: treatment of tardive syndromes: report of the Guideline Development Subcommittee of the American Academy of Neurology. Neurology 2013;81:463-9. https://doi.org/10.1212/WNL.0b013e31829d86b6

16. Mentzel CL, Bakker PR, van Os J, Drukker M, Matroos GE, Hoek HW, et al. Effect of antipsychotic type and dose changes on tardive dyskinesia and parkinsonism severity in patients with a serious mental illness: the Curacao extrapyramidal syndromes study XII. J Clin Psychiatry 2017;78:e279-85. https://doi.org/10.4088/JCP.16m11049
17. Niemann N, Jankovic J. Treatment of tardive dyskinesia: a general overview with focus on the vesicular monoamine transporter 2 inhibitors. Drugs 2018;78:525-41. https://doi.org/10.1007/s40265-018-0874-x

18. Bhidayasiri R, Jitkritsadakul O, Friedman JH, Fahn S. Updating the recommendations for treatment of tardive syndromes: a systematic review of new evidence and practical treatment algorithm. J Neurol Sci 2018;389:67-75. https://doi.org/10.1016/j.jns.2018.02.010

19. Jankovic J. An update on new and unique uses of botulinum toxin in movement disorders. Toxicon 2018;147:84-8. https://doi.org/10.1016/j.toxicon.2017.09.003

20. Macerollo A, Deuschl G. Deep brain stimulation for tardive syndromes: systematic review and meta-analysis. J Neurol Sci 2018;389:55-60. https://doi.org/10.1016/ j.jns.2018.02.013

21. Asser A, Taba P. Psychostimulants and movement disorders. Front Neurol 2015;6:75. https://doi.org/10.3389/ fneur.2015.00075

22. Lappin JM, Darke S, Farrell M. Methamphetamine use and future risk for Parkinson's disease: evidence and clinical implications. Drug Alcohol Depend 2018:187:134-40. https://doi.org/10.1016/j.drugalcdep.2018.02.032

\section{FURTHER READING}

Jamshidi N, Dawson A. The hot patient: acute drug-induced hyperthermia. Aust Prescr 2019;42:24-8. https://doi.org/ 10.18773/austprescr.2019.006 